\title{
Upper and lower limb muscle atrophy in critically ill patients: an observational ultrasonography study
}

\author{
Nobuto Nakanishi ${ }^{1}$, Jun Oto ${ }^{2}$, Rie Tsutsumi ${ }^{3}$, Marina luchi ${ }^{3}$, Mutsuo Onodera ${ }^{4}$ and Masaji Nishimura ${ }^{4 *}$ (i)
}

\section{Dear Editor,}

Skeletal muscle weakness and physical disability are common in critically ill patients. After ICU admission, noticeable reduction in muscle mass and attendant functional disabilities start within 3 days and thereafter worsen progressively [1]. Ultrasonography is noninvasive and easily available at the bedside, and a useful tool for evaluating muscle atrophy [2]. Typically, ultrasonographic assessment of muscle mass is carried out on lower limbs. Faced with limited and seemingly anomalous data for upperlimb muscle atrophy in bed-ridden patients [3, 4], we investigated whether critical illness was associated with upper-limb muscle atrophy. The study was approved by the clinical research ethics committee at Tokushima University Hospital (approval number 2593). At enrolment, written informed consent was obtained from patients or from an authorized surrogate.

We consecutively recruited adult patients who were expected to require mechanical ventilation for longer than $48 \mathrm{~h}$ and to remain in the ICU more than 5 days. All scanning was done with patients in supine and elbows and knees in passive extension. The transducer was placed perpendicular relative to the long axis of the limbs. The muscle mass of the biceps brachii and rectus femoris were evaluated on days $1,3,5$, and 7 with serial ultrasonagraphic measurements of thickness and crosssectional area.

Twenty-eight patients were enrolled, and all patients remained in the study on day 3,23 on day 5 , and 21 on

\footnotetext{
*Correspondence: nmasaji@tokushima-u.ac.jp

${ }^{4}$ Emergency and Critical Care Medicine, Tokushima University Graduate School of Biomedical Sciences, 3-18-15 Kuramoto, Tokushima 770-8503, Japan

Full author information is available at the end of the article
}

day 7. The mean age was $68 \pm 9$ years, 18 males, and median APACHE II score 27.5 (23.0-29.3). Biceps brachii thickness and cross-sectional area decreased by $6.5,11.0$, and $13.2 \%(p<0.01)$, and by $8.3,11.1$ and $16.9 \%$ on day $3,5$, and $7(p<0.01)$, respectively (Fig. 1$)$. Rectus femoris thickness and cross-sectional area decreased by 7.4, 11.1, and $18.8 \%$, and by $8.7,13.7$, and $20.7 \%$ on days 3,5 , and 7 $(p<0.01)$, respectively. Intra- and inter-observer reproducibility was $0.96-0.99$ and $0.98-0.99$, respectively.

Turton et al. found that the muscle thickness of the upper limbs of ICU patients remained unchanged during the first 10 days, while APACHE II score was lower and their patients were younger than in the present study [3]. de Boer et al. investigated voluntarily immobilized normal patients [4]. Healthy volunteers used their arms actively during bed rest, and this may have counteracted the tendency to atrophy. Measurement protocols may also be attributable to the disparity between our results and previous studies. We measured both muscle thickness and cross-sectional area, and regard our measurement as more precise and accurate, or, at the very least, less susceptible to measurement bias [5].

This study is limited by the small sample size. Meanwhile, we were unable to evaluate muscular strength and function. We still do not know to what extent muscle atrophy can be reversed; inability to restore muscle mass, especially in elderly patients, to previous levels can negatively affect patient long-term outcome.

Our findings show both upper and lower limbs wasted in critically ill patients. It is prudent to monitor upperlimb muscle atrophy as well as lower-limb muscle atrophy.

\section{Springer}




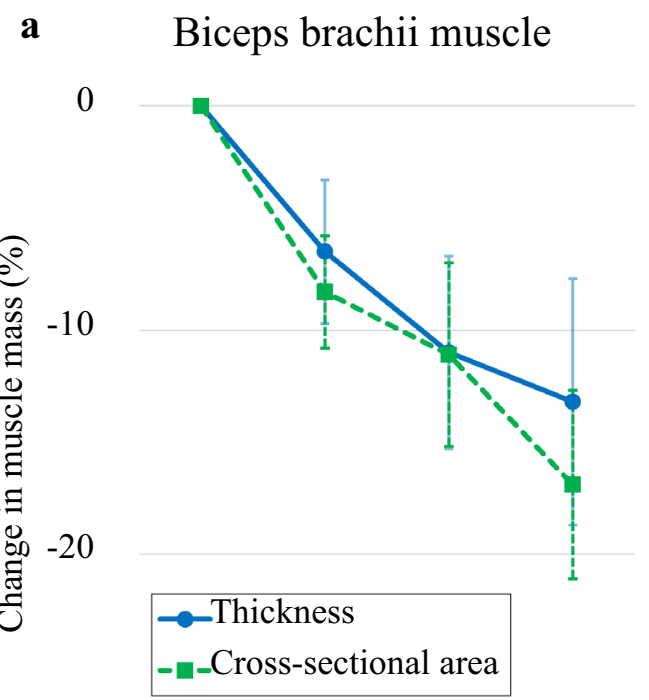

$-30$

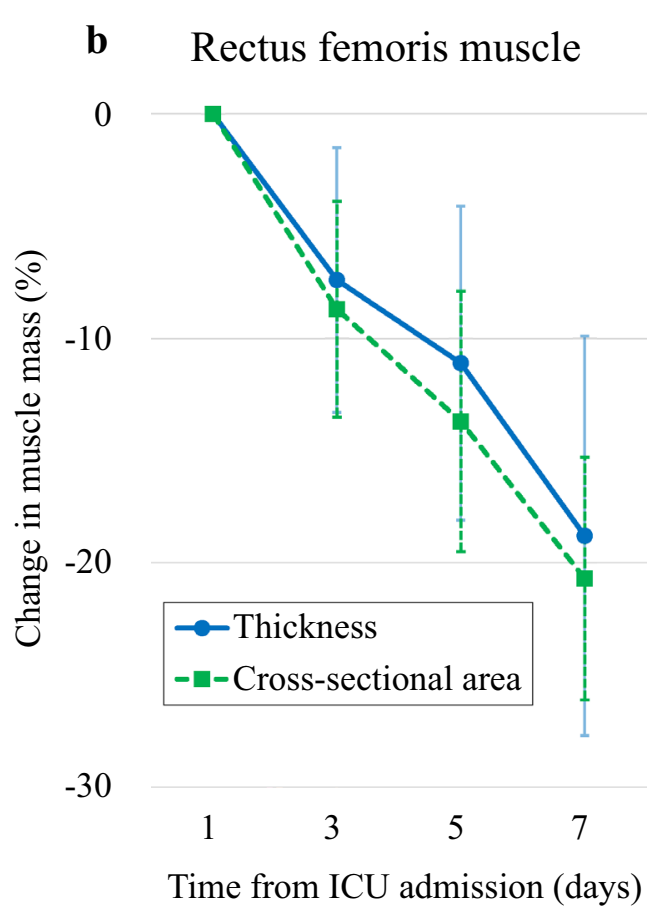

Fig. 1 Change in muscle mass of biceps brachii and rectus femoris. Muscle mass of both biceps brachii and rectus femoris progressively decreased. a Biceps brachii thickness and cross-sectional area statistically significantly $(p<0.01)$ decreased. b Rectus femoris thickness and cross-sectional area statistically significantly $(p<0.01)$ decreased. $p$ values were derived from a generalized linear mixed model. Data are expressed as means and $95 \%$ confidence intervals. CSA cross-sectional area

\section{Electronic supplementary materials}

The online version of this article (https://doi.org/10.1007/s00134-017-4975-x) contains supplementary material, which is available to authorized users.

\section{Author details}

${ }^{1}$ Emergency and Critical Care Medicine, Tokushima University Hospital, 2-50-1 Kuramoto, Tokushima 770-8503, Japan. ${ }^{2}$ Emergency and Disaster Medicine, Tokushima University Hospital, 2-50-1 Kuramoto, Tokushima 770-8503, Japan. ${ }^{3}$ Department of Nutrition and Metabolism, Tokushima University Graduate School of Biomedical Sciences, 3-18-15 Kuramoto, Tokushima 770-8503, Japan. ${ }^{4}$ Emergency and Critical Care Medicine, Tokushima University Graduate School of Biomedical Sciences, 3-18-15 Kuramoto, Tokushima 770-8503, Japan.

\section{Open Access}

This article is distributed under the terms of the Creative Commons Attribution-NonCommercial 4.0 International License (http://creativecommons.org/ licenses/by-nc/4.0/, which permits any noncommercial use, distribution, and reproduction in any medium, provided you give appropriate credit to the original author(s) and the source, provide a link to the Creative Commons license, and indicate if changes were made.

Accepted: 21 October 2017

Published online: 6 November 2017

\section{References}

1. Puthucheary ZA, Rawal J, McPhail M, Connolly B, Ratnayake G, Chan P, Hopkinson NS, Phadke R, Dew T, Sidhu PS, Velloso C, Seymour J, Agley CC, Selby A, Limb M, Edwards LM, Smith K, Rowlerson A, Rennie MJ, Moxham J, Harridge SD, Hart N, Montgomery HE (2013) Acute skeletal muscle wasting in critical illness. JAMA 310:1591-1600

2. Bunnell A, Ney J, Gellhorn A, Hough CL (2015) Quantitative neuromuscular ultrasound in intensive care unit-acquired weakness: A systematic review. Muscle Nerve 52:701-708

3. Turton P, Hay R, Taylor J, McPhee J, Welters I (2016) Human limb skeletal muscle wasting and architectural remodeling during five to ten days intubation and ventilation in critical care - an observational study using ultrasound. BMC Anesthesiol 16:119

4. de Boer MD, Seynnes OR, di Prampero PE, Pisot R, Mekjavic IB, Biolo G, Narici MV (2008) Effect of 5 weeks horizontal bed rest on human muscle thickness and architecture of weight bearing and non-weight bearing muscles. Eur J Appl Physiol 104:401-407

5. Puthucheary ZA, McNelly AS, Rawal J, Connolly B, Sidhu PS, Rowlerson A, Moxham J, Harridge SD, Hart N, Montgomery HE (2017) Rectus femoris cross-sectional area and muscle layer thickness: comparative markers of muscle wasting and weakness. Am J Respir Crit Care Med 195:136-138 